\title{
Die Reporterin Alice Schalek bei der Isonzoarmee
}

Und hier in dieser unvergeßlichen Minute, da ich zum ersten Mal am Ufer des Isonzo stehe, jenes Flusses, der uns zum Ehrenzeichen geworden ist, der einen Schlachtruf, ein Idol für Tausende bildet, hier zwischen den gestorbenen Häusern und angesichts der zu Wohnungen gewordenen Erdlöcher, erscheint mir plötzlich der Krieg in seiner ganzen unsäglichen Grausamkeit. Gibt es einen, der fassen kann, warum man die Häuser zerschießt und die Menschen in Felshöhlen treibt? Wer fordert das, was doch keiner will? Der Krieg? Wer ist dieser Krieg? ${ }^{1}$

Die Berichterstattung Alice Schaleks über den Ersten Weltkrieg wird in diesem Beitrag einer kritischen Analyse unterzogen, wobei der Nachweis erbracht wird, dass es sich in ihren Artikeln nicht nur um Propaganda handelt, sondern dass die Autorin über die Isonzo-Schlachten kritisch berichtet, indem sie sowohl das Kriegsgeschehen, seine Folgen für das Heer und die Zivilbevölkerung schildert als auch eigene Reflexionen dazu wiedergibt. Bis zum heutigen Tag ist nämlich gerade in ihrem Fall bei der Analyse der Berichterstattung eine den Ausführungen von Karl Kraus einseitig und mitunter unkritisch folgende Orientierung zu beobachten. Es wird von Schalek mehr geredet als von ihr gelesen.
Im Beitrag wird die Berichterstattung von Alice Schalek über die Schlachten am Isonzo thematisiert. Dabei gelangt die Verfasserin zu der Überzeugung, dass die einzige Berichterstatterin im Ersten Weltkrieg einerseits Propaganda im Sinne der Habsburger Monarchie betreibt, dabei ihren niedrigsten Instinkten frönend (K. Kraus), zugleich aber die vielen anonym Kämpfenden der Vergessenheit entreißt. 


\section{Biografisches}

Die Journalistin, Fotografin und Autorin Alice Therese Emma Schalek (geb. 1874 in Wien, gest. 1956 in New York City) stammte aus einer bürgerlichen jüdischen Familie. Unter dem männlichen Pseudonym Paul Michaely veröffentlichte sie 1902 den Roman Wann wird es tagen? Sie wurde 1903 als Journalistin im Feuilleton der »Neuen Freien Presse« in Wien aktiv, für die sie über 30 Jahre lang tätig war. Auch sie konvertierte, genauso wie der ihr gegenüber kritische Kollege Karl Kraus.

Gleich zu Beginn des Krieges, am 31. August 1914, wurde Schalek zur Mitgründerin der Wohltätigkeitsorganisation Schwarz-gelbes Kreuz, die sich um Kriegsinvaliden und ihre Angehörigen kümmern wollte. Auf ihren Wunsch wurde sie 1915, damals bereits 41-jährig, als Kriegsberichterstatterin zugelassen und beim k.u.k. Kriegspressequartier in Österreich akkreditiert. Sie berichtete über die Gebirgsschlachten in den Dolomiten, über den Serbien-Feldzug, die Isonzo-Front, die Verhältnisse an der herzegowinischen und montenegrinischen Grenze und an der Adria. Ihre Artikel stießen in der Öffentlichkeit auf ein geteiltes Echo. Unter den Berufskollegen gehörte Karl Kraus zu ihren schärfsten Kritikern. Er warf ihr Kriegsverherrlichung vor und stellte ihre Arbeit in seinem Drama Die letzten Tage der Menschheit kritisch dar. ${ }^{2}$ Schaleks Bruder Norbert, der Oberleutnant war, forderte ihn nach der Veröffentlichung der 30. Szene des II. Aktes (Irgendwo an der Adria) und des Kommentars zur Berichterstattung seiner Schwester über den serbischen Feldzug zum Duell. Der Publizist verwies auf seinen Rechtsanwalt, worauf Alice Schalek eine Beleidigungsklage gegen ihn erhob, ${ }^{3}$ die sie 1917 zurückzog. In diesem Jahr beendete sie auch ihre Tätigkeit als Kriegsreporterin.

Von den Kriegsschauplätzen berichtete sie in ihren Zeitungsartikeln oder Büchern und hielt über das Erlebte Vorträge, die die Säle füllten. Dass diese Tätigkeiten im Sinne der offiziellen Politik durchgeführt wurden, steht außer Zweifel. Und doch sind ihre Aufsätze und Vorträge nicht ausschließlich als Propaganda, sondern auch als sachliche Berichte zu bewerten, die

2 Kraus: Die letzten Tage der Menschheit.

3 Darüber berichtete auch das Laibacher Tagesblatt „Slovenski narod « in seiner allen sichtbar zensierten Rubrik Tagesneuigkeiten, indem an ihren Laibacher Vortrag angeknüpft wurde. Das Blatt informierte seine Leserschaft darüber, dass Schalek in Wien an einer Verhandlung teilnehmen würde, weil sie »den bekannten Wiener Autor Karol Kraus« (Hervorhebung M. M. Z.) wegen Verunglimpfung ihrer Person »angeklagt habe«. Kraus, so das Blatt, habe sich über ihre Berichterstattung zur Einnahme Belgrads und der Lage am Isonzo schrecklich mokiert. Vgl.: Anonym: Alice Schalek (3.11.1916), S. 3. 
das Leben der Soldaten und der in Mitleidenschaft gezogenen Bevölkerung wiedergeben. Und sie fielen auf - nicht nur unangenehm. Am 18. Februar 1917 wurde sie, ungewöhnlich für eine Frau, mit dem Goldenen Verdienstkreuz mit der Krone am Bande der Tapferkeitsmedaille »[i]n Anerkennung vorzüglicher und aufopferungsvoller Leistungen als Kriegsberichterstatterin « mit der nachfolgenden Begründung ausgezeichnet: »Hat in ihrer Eigenschaft als Berichterstatterin für die `Neue Freie Presse ‘ bei Görz und an der gesamten Isonzofront Eindrücke gesammelt, hiebei das feindliche Feuer nicht gescheut und dank ihrer literarischen Begabung die Isonzoarmee durch eine Serie gehaltvoller Publikationen popularisiert. ${ }^{4}$

Karl Kraus, Zeitgenosse und schärfster Kritiker der Alice Schalek, nannte sie eine »Jourjüdin«, ein »Monstrum«, »eines der ärgsten Kriegsgreuel«, und bezeichnete ihre Front-Berichte als

das Schauspiel einer Entartung, das unsere besondere kulturelle Situation als eine vor dem übrigen Europa avancierte zeigt. Denn es ist möglich geworden [...] daß unsere Öffentlichkeit die obszönen Tagebuchblätter vorgesetzt bekommt, die ein Frauenzimmer verfaßt hat, das sich für seine Weiblichkeit kein anderes Feld der Anregung zu verschaffen wußte als das Feld der Ehre - ausgerechnet! Pfui Teufel! ${ }^{5}$

Hart und schonungslos. Dabei dürfte es 1916 allgemein bekannt gewesen sein, dass das Oberarmeekommando seine Berichterstatter nur dann über die Front informieren ließ, »wenn sie über einen Erfolg berichten konnten « ${ }^{6}$ Hatten Kraus, neben seiner Frauen- und Judenfeindlichkeit, zu dieser Aussage und weiteren ähnlich gehässigen Äußerungen etwa jene Stellen der Berichte der einzigen Berichterstatterin aus dem Ersten Weltkrieg bewogen, in welchen Schalek persönlicher wurde, den Leser zum Mitwisser machte und sich als eine eitle Person zeigte?

\footnotetext{
Weil mich Cadorna heute wiederum verschonte, weil die Granate wiederum gerade um ein Viertelstündchen zu spät kam, gibt's eine Flasche echten Champagners und als besonderen Lohn eine Dose wirklichen Kaviars. Knusprige Kipfel und bunte Blumen, Radieschen und ein Damastgedeck - solche Kontraste gibt es nur an der Front. ${ }^{7}$
}

Oder waren der Grund seiner Attacken eher jene Passagen, in denen sie das ganze Elend eines Stellungskrieges in einem Tableau erfasst, in welchem, beispielsweise, der einstige Verbündete Italien, mit dem man vor dem Krieg eine gemeinsame Flottenstrategie entwickelt hatte und die eigene Kriegs- 
marine als Fleet-in-Being ausbauen ließ, ${ }^{8}$ zum Gegner geworden war, der als `fremd ‘, sanders` und >pietätlos` dargestellt wird?

Unsere Leute, die den Sturm gestern mitmachten, sagen, eine Kloake sei ein paradiesischer Aufenthalt gegen diese italienische Isonzolinie. Natürlich verschlimmert es den Zustand, daß die Italiener von Natur aus unrein sind [...] Oft und oft wird mir erzählt, daß sie gar keine Latrinen haben. Auch fehlt ihnen jede Pietät für die Toten. Zahllose italienische Leichen liegen überall vor ihrer Front.'

Wir können das heute nicht mit Sicherheit feststellen.

\section{Propaganda}

Im Bezug auf Kriege stellt sich immer öfter die Frage, welche Rolle des gesprochenen und geschriebenen Wortes geübte Intellektuelle, Schriftsteller, Reporter, Publizisten oder Journalisten, also auch Alice Schalek im Großen Krieg, gespielt haben: Haben sie den Krieg verherrlicht und propagiert oder dagegen protestiert? ${ }^{10}$

Als es 1914 zum Krieg kam, wunderte das kaum jemanden, wohl aber dachten viele, er würde von kurzer Dauer sein. Um ihn zu feiern, fehlte es nicht an begeisterten Dichtern, Malern, Bildhauern und Musikern, ${ }^{11}$ die sich entweder freiwillig zum Kampf meldeten oder den Krieg wie Ernst Jünger ${ }^{12}$ oder Thomas Mann (Pflichtgefühl) verherrlichten. Es gab auch einige wenige, die von Anfang an dagegen waren und dies nicht verschwiegen, wie beispielsweise Johannes R. Becher, Annette Kolb, Ricarda Huch, Arthur Schnitzler, Leonhard Frank und Heinrich Mann. Karl Kraus aber, der gegen >die < Schalek so wortmächtig und effektiv anschrieb, meldete sich zum Attentat in Sarajevo erst am 10. Juli 1914 mit dem Artikel Franz Ferdinand und die Talente. Danach schwieg er bis Dezember 1914, was sich zwischen Ende Februar und Anfang Oktober 1915 wiederholen sollte:

Was aber das lange Schweigen verursacht hatte, war wirklich ungewöhnlich. Um sich von der brüderlichen Bevormundung zu befreien, entschloß sich Sidonie Nadherny [die große Liebe von K. Kraus], den italienischen Grafen Carlo Guicciardini zu heiraten, und fuhr im Februar 1915 nach Rom. Karl Kraus, den bisher keine persönlichen Erlebnisse [...] von der Arbeit abhalten konnten, warf alles hin, um ihr zu folgen. [...] Am 24. kehrte

8 Rauchensteiner: Der Erste Weltkrieg, S. 266-269.

9 Schalek: Bei der Isonzoarmee. Ein Angriff über die Tolmeiner Brücke, S. 4.

10 In diesem Zusammenhang seien hier nur zwei Publizistinnen genannt: Bertha von Suttner und Alice Schalek, wobei Suttner eine aktive Kriegsgegnerin und Pazifistin war. Mehr zu Suttner in: Hamann: Bertha von Suttner.

11 Rauchensteiner: Der Erste Weltkrieg, S. 231.

12 Kirschstein: Writing War. 
Karl Kraus nach Wien zurück, und bald danach machte der Kriegseintritt Italiens an der Seite der Alliierten die Hochzeitsvorbereitungen endgültig zunichte. Einen Sommermonat verbrachte Karl Kraus mit Sidonie Nadherny in der Schweiz, und dort faßte er den Entschluß, sein Schweigen zu brechen [...] Im Oktober 1915 erschien ein Heft der Fackel [...]. ${ }^{13}$

Erst dann wurde er zu einem heftigen Gegner des Krieges und schrieb so scharf dagegen an, wie es die Zensur eben zuließ:

Karl Kraus war in diesem Chaos einer der wenigen Menschen, die unerschrocken und klar aussprachen, was alle diese Greuel, diese zur Pflicht gewordene Bestialität zu bedeuten habe; er hat diejenigen ernüchtert, die sich von der Begeisterung der ersten Kriegsmonate hatten verblenden lassen, und er hat die Verzweifelnden alle getröstet und aufgerichtet und einander wissen lassen, daß diese Einzelnen wenigstens das Band des Geistes und des Glaubens verbindet. ${ }^{14}$

Der Erste Weltkrieg bedeutet bezüglich Propaganda auch in der Habsburger Monarchie einen markanten Wendepunkt, da man zur Informierung und Beeinflussung der Bevölkerung zu aktuelleren und subtileren Mitteln griff als bis dato. Neben der alterprobten Presse, neben mehrsprachigen Plakaten und Ansichtskarten mit teilweise vorabgedruckten Text, neben der wissenschaftlichen und schöngeistigen Literatur, den Bildern, Zeichnungen und Skulpturen, bediente man sich nämlich auch des Theaters, des Funks und des Films. ${ }^{15}$ Bekanntlich wurden kurz nach Ausbruch des Krieges allein im Deutschen Reich täglich bis zu 50.000 vaterlandsliebende Gedichte an Zeitungen geschickt, die meisten davon bereits aus dem Feld. ${ }^{16}$ Nicht viel anders verhielt es sich bei den Alliierten und auch nicht im slowenischen ethnischen Gebiet, wie das Gedicht eines Isonzo-Soldaten des k.u.k. Infanterieregiments Kronprinz Nr. 17 zeigt: »Slednji polk med sabo / v hrabrosti tekmuje, / izmed vseh se `kranjski /Janez` odlikuje./ Kjer šrapnelov, krogel / dež se gosti vsuje, / kjer nevihta bojna / zadivja najhuje, / tja hitijo Kranjci / na usodno mesto, / kot neustrašni levi / se borijo zvesto « ${ }^{17}$ Man engagierte zu diesem Zweck auch arrivierte Autoren, Maler, Bildhauer und Journalis-

Schick: Karl Kraus in Selbstzeugnissen und Bilddokumenten, S. 77f.

14 Liegler: Karl Kraus und sein Werk, zit. nach Schick: Karl Kraus in Selbstzeugnissen und Bilddokumenten, S. 79.

15 Die Propaganda wurde von der Politik und vom Militär betrieben. Dabei verzichtete man noch auf die Hilfeleistung von Marketing-Experten, was sich im Zweiten Weltkrieg und danach grundlegend ändern würde, nicht zuletzt durch die schnelle Entwicklung der elektronischen Medien und ihren Möglichkeiten.

16 Kraus versus Schalek.

17 Štepec (Hg.): Slovenci + prva svetovna vojna 1914-1918, S. 13. In dürftiger dt. Übersetzung: "Jedes Regiment konkurriert mit anderen in Tapferkeit, vor allen zeichnet sich der >Krainer Johann ‘ aus. Wo der Schrapnellen, Kugeln dichter Regen niederfällt, wo das Kampfesgewitter am wildesten lodert, dahin eilen die Krainer zum schicksalsträchtigen Ort, treu kämpfend wie unerschrockene Löwen.« 
ten, die zum Aufbau der Moral im Allgemeinen und zur Unterstützung der Truppen im Besonderen beizutragen hatten. Zu letzterer gehörten ebenfalls die nicht seltenen Truppen-Besuche des letzten österreichischen Kaisers Karl samt Gattin Zita an der Front, auch am Isonzo, ${ }^{18}$ oder im Hinterland.

Die Propagandamaschinerie ging in ihren Bemühungen, die Öffentlichkeit von der Gerechtigkeit der eigenen Ansprüche und der Notwendigkeit des Krieges zu überzeugen, noch einen Schritt weiter. Sie bereitete die Bevölkerung mitunter schon vor dem Krieg auf künftige Kämpfe vor, dabei auf edle Gefühle wie Patriotismus appellierend. So geschehen vor der Kriegserklärung Österreich-Ungarns an Serbien, als das erfundene Gefecht bei Kubin an der Donau als Überfall serbischer auf die österreichischungarischen Truppen dargestellt wurde; diese Falschmeldung fand Eingang in den Text der Kriegserklärung. ${ }^{19}$

Auf ein erdichtetes Gefecht stützte sich also die Kriegserklärung an Serbien, die sich bald zu einer regelrechten Inflation von Kriegserklärungen auswachsen sollte. Die Lage in der Habsburger Monarchie war damals in Kürze, wie folgt: Der slawenliebende, reformlustige und zuhause eher unbeliebte Thronfolger wurde ermordet. Die österreichischen Generäle, die alt waren und sich einen Namen als Manövergeneräle ${ }^{20}$ gemacht hatten, wurden, wohl um den Feind zu täuschen, einen Monat vor der Kriegserklärung in den Urlaub geschickt, aus dem sie am 25. Juli zurückzukehren hatten. Franz Joseph, getragen von der Überzeugung, Serbien liege wegen der vorausgegangenen Balkankriege am Boden zerstört, was nicht ganz der Fall war, ${ }^{21}$ wollte dem südslawischen Land eine denkwürdige Lektion erteilen, nicht aber einen Krieg von Weltausmaßen anfeuern. Dabei vergaß er, genauso wie sein Heer, dass die Donau-Monarchie nicht über die modernsten Waffen verfügte, abgesehen vielleicht von der Flotte, die dahingehend ausgerichtet war, im Dreibund mit Italien (das nach Kriegsausbruch fast ein Jahr lang neutral blieb, um sich danach gegen Österreich-Ungarn zu wenden) und Deutschland zu wirken. Die modernen Waffen waren vor dem Krieg exportiert worden, teilweise an die späteren Gegner, ${ }^{22}$ so dass

Darüber wurde auch in der »Laibacher Zeitung « berichtet. Vgl.: »LZ«, 12.4.1917, S. 542; »LZ«, 13.4.1917, S. 547.

19 Vgl. Rauchensteiner: Der Erste Weltkrieg, S. $129 f$.

20 Vgl. ebd., S. 240. Äußerst treffend werden Manöveroffiziere in Joseph Roths Roman Radetzkymarsch dargestellt.

21 Das serbische und das russische Heer waren, seinen Erfahrungen in den beiden Balkankriegen zufolge, anfänglich die zwei einzigen Armeen mit einer zeitgemäßen Kriegsführungsart. Die serbische Armee war außerdem mit modernen Waffen ausgerüstet. Vgl. Fernsehsendung $V$ fokusu.

22 Rauchensteiner: Der Erste Weltkrieg, S. 46f. 
man die drei Millionen zusätzlich Mobilisierten mit den eigenen modernen Waffen nicht zum richtigen Zeitpunkt ausrüsten konnte. Auch infolge dieser Falscheinschätzungen breitete sich, sozusagen unversehens, ein Großer Krieg aus, ${ }^{23}$ der ob seiner Länge und Gründlichkeit alle überraschte und sich schnell zu einem »totalen Krieg ${ }^{24}$ entwickelte.

Kaum jemandem, ausgenommen Bertha von Suttner, war es vor dem Krieg so richtig bewusst, dass in der ganzen alten und neuen Welt eine Kultur des Kriegs herrschte. ${ }^{25}$ Und das war auch selbst dann, als man am Krieg schwer zu tragen hatte und sich im Klaren über die ganzen Ausmaße der Gemetzel und Aushebungen von Geiseln war, niemandem bewusst, außer der im Krieg dienenden Soldaten oder eben einer Berichterstatterin wie Schalek:

Ein Erfolg! Die ganze Monarchie wird das morgen zum Abendbrot lesen, überall werden die Gläser glückselig geleert. Erfolg! Ich aber denke stumm an die Telephonisten, an die Minenwerfer, an die Sanität. Mir scheint er blutigrot, dieser Erfolg. »Wir haben nur geringe Verluste.« Und in dem zugewiesenen Quartier, dem ersten seit vierzehn Tagen, das keine Granate bedroht, sitze ich lange und weine. ${ }^{26}$

Gewiss wurde dieser Krieg auch inszeniert und manipuliert, worauf Kraus ebenfalls hinwies und damit Schalek kritisierte. Er verwies dabei auf Stellen ihrer Berichterstattung wie diese:

Alle Herren sind zu unserem Empfange oben versammelt. Sonst hockt jeder wohlgedeckt oder er schläft, jedenfalls hütet er sich sehr, hier offen spazieren zu gehen. Aber weil der erste Kriegsberichterstatter angekündigt worden ist, sitzen die Herren gemütlich wie im Rathauskeller beisammen und erwarten uns. ${ }^{27}$

23 Das österreichische Heer zählte in der Friedenszeit 1,5 Millionen Mann. Im Juli 1914 wurden weitere 3 Millionen mobilisiert, davon 2 Millionen in den Krieg geschickt. Im Karpatenwinter 1914/15 fielen 1,5 Millionen Mann aus: gefallen, vermisst, verletzt oder gefangen genommen. Vgl. Fernsehsendung $V$ fokusu.

24 Rauchensteiner: Der Erste Weltkrieg, S. 220.

25 Die Bemühungen der aktiven Pazifistin und Publizistin Bertha von Suttner (1843-1914) um den Weltfrieden stießen bei ihren Zeitgenossen nicht nur auf Unverständnis, sondern auch auf Spott und Diffamierung. Der ihr 1905 verliehene Friedens-Nobelpreis konnte daran wenig ändern. Mehr dazu in: Lughofer: Die Waffen nieder! aus interkultureller Perspektive; Wintersteiner: "Die Waffen nieder!« - ein immer noch zeitgemäßes Projekt; Miladinović Zalaznik: Bertha von Suttners Die Waffen nieder!

26 Schalek: Bei der Isonzoarmee. Von der Front in die Etappe, S. 2.

27 Schalek, Alice: Bei der Isonzoarmee. Eine Mondnacht auf dem Monte Sabotino, S. 2 (Hervorhebung M. M. Z.); Kraus: Notizen, S. 19. In ihrem Werk Am Isonzo. März bis Juli 1916 hat Schalek die betreffende Stelle leicht abgeändert: »Alle Herren sind oben versammelt. Sonst hockt jeder wohlgedeckt in seinem Loch oder schläft, jedenfalls hütet er sich sehr, hier offen spazieren zu gehen. Aber weil der erste Kriegsberichterstatter angekündigt worden ist, sitzen die Herren gemütlich beisammen und erwarten uns.« (S. 37f.) 
Das Schalek-Zitat in der »Fackel« endet mit dem folgenden Satz:: »Man hat mit der Beschießung gewartet, bis wir oben angelangt sind, weil sonst das >Vergeltungsschießen ‘ uns den Weg recht unangenehm hätte gestalten können ${ }^{28}{ }^{8}$ Kraus kommentiert das folgendermaßen: »Man - hat gewartet! $\aleph^{29}$ Angesichts dieser Zustände ist naheliegend zu fragen, ob es am Isonzo um einen Operettenkrieg ging? Die Antwort lautete schon damals: mitnichten. Der `Löwe vom Isonzo<, wie man den General und Kommandanten der Isonzoarmee Svetozar Boroević von Bojna $(1856-1920)^{30} \mathrm{zu}$ bezeichnen pflegte, scheute in den Gefechten um Isonzo keine Opfer. Unter ihm fielen an die 160.000 Kämpfende. Und wenn man diese Zahlen 19151917 nicht gekannt haben sollte, so wusste man ganz genau, nicht zuletzt durch Schalek, dass die Schlachten am Isonzo ausnehmend blutig waren.

Ja, es wurde in diesem Krieg Propaganda getrieben und es wurde manipuliert, auf allen Seiten. Als es den italienischen Einheiten während der dritten Isonzo-Schlacht im Oktober/November 1915 gelungen war, den strategisch wichtigen Gipfel Mrzli Vrh (1359 m) für einen Tag einzunehmen, hörte man in italienischer Presse nicht auf, über diesen Sieg am Isonzo zu berichten, als wäre er von längerer Dauer gewesen. ${ }^{31}$ Der italienische General Cadorna und der k.u.k. General von Boroević erlitten dabei große Verluste, befahlen jedoch ihren Einheiten, nicht zu weichen. ${ }^{32}$

\section{Am Isonzo}

Schalek verbrachte die Monate März bis Juli 1916 an der Isonzo-Front, die sie sowohl in ihren Artikeln als auch in dem im gleichen Jahr herausgebrachten, dem General von Boroević gewidmeten und 1917 in einer zweiten Auflage erschienen Werk in Schrift und Bild dokumentierte: Am Isonzo. März bis

Kraus: Notizen, S. 19. Diese Stelle hat er ihrem Artikel entnommen: Schalek, Alice: Bei der Isonzoarmee. Eine Mondnacht auf dem Monte Sabotino, S. 3; auch diese Passage hat Schalek in ihrem Buch leicht abgeändert, und zwar folgendermaßen: »Man hat mit der Beschießung gewartet, bis der Generalstabschef oben angelangt war, weil sonst das >Vergeltungsschießen $<$ seinen Weg recht unangenehm hätte gestalten können. «Schalek: Am Isonzo, S. 40 (Hervorhebungen M. M. Z.).

29 Kraus: Notizen, S. 19.

30 Schaleks Interview mit Boroević, das sie in ihren Artikel vom 4.4.1916 in der »Neuen Freien Presse « eingearbeitet hatte (S. 1-3), druckten die slowenische Zeitschrift "Edinost» in Triest am 6.4.1916 und das Laibacher »Slovenski narod « am 8.4.1916 ab.

31 Popov: Sledi časa.

32 Da die Italiener massenweise desertierten, fällte Cadorna 750 Todesurteile über die eigenen Soldaten. Er hatte 1917, wie aus den bisher nicht veröffentlichten italienischen Archivdokumenten hervorgeht, von seinen Einheiten verlangt, sie sollten auf die eigenen Leute (notfalls mit Kanonen) von hinten schießen, um sie zum Kampf zu bewegen (ebd.). 
Juli 1916. Mit 109 Abbildungen meist nach eigenen Aufnahmen und einer Übersichtskarte. Sie veröffentlichte im Morgenblatt der »Neuen Freien Presse« in der Zeit vom 4. April bis zum 15. September 1916 zwanzig Berichte von der Isonzo-Front. Ihr erster Bericht wurde genau zwei Monate später, am 4. Juni 1916, in voller Länge noch einmal gedruckt. Am 12. und am 15. Juni 1917, ein Jahr nach der zehnten Isonzoschlacht, wurden ihre beiden Artikel zu dieser Schlacht (Bilder von der zehnten Isonzoschlacht. Die Kote* und Bilder von der zehnten Isonzoschlacht. Die Kote ${ }^{* *}$ ) ebenfalls noch einmal publiziert. Alle drei Nachdrucke erfolgten ohne nähere Begründung.

Ihren ersten Bericht vom Isonzo, der, wie alle anderen auch, »[v]om Kriegspressequartier genehmigt « wurde und laut einem Vermerk nicht nachgedruckt werden durfte, ${ }^{33}$ beginnt Schalek mit einer kleinen Stimmungsszene, einem Tableau, das sie in einer Wiener Buchhandlung situiert. In den Eingangssätzen formuliert sie ihr Berufscredo:

Wozu vom Kriege auch noch lesen? Hört man nicht genug von ihm? [...] Nein, ich meine man hört nicht genug, weiß viel zu wenig von ihm. Die furchtbar knappen Worte, in die man ihn für uns zusammengepreßt hat, stehen wie eine Mauer vor uns: Schlacht! - Welch ein Ausdruck! Sieg! - Welch eine Zusammenfassung! Daß dahinter Menschen sterben wissen wir's? Während der Krieg Hunderttausende von Leben umkrallt und mitten aus ihnen heute den - morgen jenen verbluten läßt - sollen wir uns da die Ohren zuhalten, damit wir ungestörter lachen oder träumen können, damit wir sagen können: »Bitte, nichts vom Krieg? « ${ }^{34}$

Das Anliegen der Berichterstatterin besteht darin, dem unbekannten, wortlos kämpfenden Mann eine Stimme zu verleihen, auf dass er nicht ungehört und unbemerkt durch die Kämpfe hindurch und aus ihnen, womöglich sogar aus dem Leben, tritt. Sie dokumentiert dabei ihre Bewunderung für Menschen, die den Krieg unter unmöglichsten Verhältnissen auszuhalten und zu gewinnen haben.

Schalek agitierte in ihren Feld-Artikeln auch, mal verhaltener, mal insistenter, für die k.u.k. Causa selbst dann, wenn sie kritisch wurde. So vergaß sie nicht, auf die Multinationalität des Heeres und das zerrüttete Verhältnis der Völker der Donau-Monarchie hinzuweisen:

Die Dalmatiner singen kroatische Lieder. Neben mir sitzt ein Vorkämpfer italienischer Sprache und Kultur. Aus vollem Halse singt er mit. "Vor zwei Jahren wäre ich jedem an die Gurgel gesprungen, der mir da mitzusingen zugemutet hätte. Jetzt singen die anderen ebensogerne meine Weisen. Warum hat erst der Krieg kommen müssen, um Oesterreicher aus uns zu machen? Und wie wird es nachher sein? «35 
Ich kann mich heute nicht festlegen, ob die nachfolgende Szene eher als Propaganda oder als erschütternder Tatsachenbericht zu bewerten ist:

Es wurde festgestellt, daß auf dem ganzen Hang des Rozmarice keine einzige Latrine war. Man fand noch 1066 unbeerdigte Leichen vor und das Grauenhafte war eine Treppe aus vierzehn Menschenleichen, die über die steilste Stelle führte. Ohne sie hätten die Alpini wohl die Bergwand nicht überwinden können [...]. ${ }^{36}$

Auch in allen anderen Berichten blieb sie ihrer Schreibweise treu: Sie schuf impressionistische Stimmungsbilder, in denen sie Görz, seine Menschen, seine Häuser, seine Hotels und seine Beschießung schilderte. ${ }^{37}$ Sie schilderte, wie man mit Batteriewagen abgeholt wurde, um die feindlichen Stellungen und die k.u.k. Nachschubwege zu studieren. Sie schilderte das Erschaute und Erfühlte: die andauernde Todesgefahr, die von den Soldaten nicht mehr wahrgenommen wurde; ihr eigenes Glück, einmal mehr unverwundet geblieben zu sein; die engen Erdlöcher, wo sich die Mannschaft versteckte; Beobachtungsstände, Kämpfe, Präzisionsschießen, Leichengeruch; eine Mondnacht auf dem Monte Sabotino, trotz Schießereien in romantischer Szenerie, wie die am Fels klebenden »Schwalbennester $«,{ }^{38}$ wo sich die Soldaten verbergen konnten; die großen Taten des kleinen Mannes, den Friedhof im Bau, ${ }^{39}$ bisweilen die eigene Arbeit mit der Kamera. Selten werden Opfer, derer Zahl in die Tausende ging, ${ }^{40}$ oder Gefangene erwähnt, ${ }^{41}$ nur einige Male der Schnee im Hochsommer, ${ }^{42}$ gegen den die Mannschaft in leichter Montur auf dem 1600 m hohen Gipfel Krn ebenfalls anzukämpfen hatte.

Zuweilen wird in ihren Reportagen vom Krieg wie von Normalität erzählt: »Ein Offizier holt mich zu[m] Besuch [Podgoras] vom Kaffeehaus ab. «3 Dort angekommen, wird das zerstörte Dorf in Augenschein genommen: »Dicht am jenseitigen Ufer liegt oder lag das Dorf Podgora mit der Papierfabrik. Wein und Oliven, Kastanien und Frühgemüse und

Schalek: Bei der Isonzoarmee. Auf den Tolmeiner Brückenkopf, den Mrzli Vrch und Bodil Vrch, S. 2.

Schalek: Bei der Isonzoarmee. In Görz.

Schalek: Bei der Isonzoarmee. Eine Mondnacht auf dem Monte Sabotino, S. 2.

»Die Gräber sind erst zur Hälfte zugeschüttet und noch ohne Schmuck.« (ebd., S. 1)

»Der Dummkopf von Cormons soll selbst herüberkommen und schauen. Hier liegen schon 6000 Tote « "In: Schalek: Bei der Isonzoarmee. Die Kote von Plava, S. 4.

1 »Am nächsten Morgen besuche ich die gefangene Mannschaft. Sie liegt in einer Holzbarracke, froh und zufrieden. Die Erlöser sind gleichsam erlöst. >Madame«, sagt mir der eine, ’wir haben drei Tage nichts gegessen. Wir steckten bis zu den Knien im Schlamm. Wir haben drei Stunden Feuertrommel ertragen. Fragen Sie uns nicht, ob wir Ihre Feinde sind. Wir sind die Geopferten «." Schalek: Bei der Isonzoarmee. Ein Angriff über die Tolmeiner Brücke, S. 3.

2 "Der Zugsführer seilte seine Leute an, damit in dem schrecklichen Schneetreiben keiner verloren gehe. "In: Schalek: Bei der Isonzoarmee. Aufstieg zum Krn, S. 2.

Schalek: Bei der Isonzoarmee. Das Geheimnis der Podgora, S. 1. 
viel Industrie gab es hier. Jetzt sieht es grausig da aus. Unter allem, was ich bisher an Kriegszerstörung gesehen, ist in Podgora die gründlichste Arbeit geschehen ${ }^{44}{ }^{4}$ geleistet von Italienern, wie sie anmerkt. Unterwegs stößt sie auf die vom Militär angebauten Felder hinter der Front:

Man fährt durch blühende Obstgärten, durch üppige Gemüsepflanzungen, an den Spargelbeeten und Artischocken, an den Radieschen und Spinatblättern, an den weißen Erbsenblüten und dem lichtgrünen Bohnengeranke der Görzer Landschaft vorbei. Fast ein Frohlocken ist in mir darüber, daß diese guten Dinge [...] nun auch auf den Tisch der armen Leutnants und Kadetten kommen [...].

Je länger Schalek an der Front ist, umso mehr offenbart sich ihr die Verwüstung ganzer Landstriche, die Abtötung der Seelen:

Ich betrachte die gelbe Erde, in der ich stecke, und sehe, daß kein Quadratfuß von ihr ohne Inhalt ist. Auf dem winzigen Flecken Raum, den ich überblicke, zähle ich bis zu Hundert Dinge, dann gebe ich's auf. [...] Ein Verbandpäckchen hängt aufgerollt bis zu meinen Füßen herab, so gelb wie der Berg. [...] Ein Spaten links, ein Gewehrkolben über mir, italienische und österreichische Patrontaschen dazwischen. Zwei Handgranaten, ein Stück Drahtverhau, ein Uniformfetzen, ein Mantelkragen. Eine blühende, gesegnete Ortschaft stand einst hier. Eine Kirche zu Ehren Gottes und ein Gasthaus zur Freude der Weltlichen. Ein Stück Altar und ein Weinfaß, das ist das einzige davon, was der tote Berg noch trägt. [...] [D]iese Erde ist gestorben und graben kann man in ihr nicht mehr. Sie ist Staub, Mehl, Schutt geworden, sie haftet nicht mehr, sie ballt sich nicht mehr, sie besitzt kein Rückgrat mehr. Wir wissen, daß der stärkste Mann, wenn er tot ist, nicht mehr zu stehen vermag. Aber daß es auch Erde gibt, die keinen Halt mehr findet, das hat erst der Krieg gezeitigt. In dieser kann man Leichen nicht bestatten, sie wirft sie aus. [...] Kein Brett findet Grund, kein Wasser fließt ab oder sickert ein, sie lehnt jede Arbeit ab. Sie ist tot. Man hat mir geraten, Formalin mitzunehmen. Ich vergaß es. Aber es tut mir nicht leid. Denn wenn einer der Mitwelt erzählen will, wie die Menschen, lebende Menschen, monatelang unter Toten hausen, dann muß er wenigstens einmal dieselbe Luft mit ihnen geatmet haben. [...] Oslavija, der tote Berg, frißt mir das Herz aus dem Leib. [...] Diesen Leichnam von einem Hügel halten wir [...] »fest in unserer Hand «. So wird's in der Geschichte stehen. Aber wer wird schildern, wie man es fertig brachte, Oslavija zu halten? ${ }^{46}$

Die Bilanz der Schlachten am Isonzo war verheerend: Die Armee ausgehungert und aufgerieben, genauso die Bevölkerung. Auf allen Seiten Deserteure und Überläufer, Geiseln und Gefangene, an Neurasthenie leidende, die, wenn Soldaten, mit Stromstößen behandelt wurden $;{ }^{47}$ Selbstverstümmelte und Selbstmörder unter Offizieren, Soldaten und in der Zivilbevölkerung, wegen Grausamkeit des Krieges, Überforderung, Verzweiflung. ${ }^{48}$ Ganze Landstriche, nicht nur Kampffelder, ausgebombt und durch sonstiges

Ebd., S. 2.

45 Schalek: Bei der Isonzoarmee. Von Görz nach Doberdo, S. 2.

46 Schalek, Alice: Bei der Isonzoarmee. Oslavija, der gestorbene Hügel, S. $3 \mathrm{f}$.

47 Rauchensteiner: Der Erste Weltkrieg, S. 243.

48 Ebd., S. 232-244. 
Kriegsmaterial devastiert. Als die ersten Kriegsheimkehrer aus dem IsonzoTal 1918 nachhause kamen, fanden sie nichts mehr vor, keine Häuser, keine Felder, keine Tiere, keine Nahrung, mitunter keine Menschen, weil diese bis nach Niederösterreich ${ }^{49}$ oder nach Süditalien umgesiedelt wurden. Einen ganzen Winter lang mussten sie sich mit wilden Pflanzen und Tieren über Wasser halten. Im Frühling waren sie froh, endlich einmal den Löwenzahn als Salat und das Taubenkropf-Leimkraut im Risotto (falls vorhanden und erschwinglich) zubereiten zu können. ${ }^{50}$ Die Zeiten, als jede Einheit seinen eigenen Anbauoffizier hatte, wurden zu einer grotesken Erinnerung; auch darüber hat Schalek berichtet:

Wie jedem Kommando an der Isonzofront ist auch diesem ein eigens beorderter Anbauoffizier zugeteilt [...] Persönlich führte er mich zu den Feldern hinaus, wo in jedem Block auf einer Tafel steht, was und wann hier angebaut worden ist. Unter der Leitung von Berufsökonomen werden Zisternen eingefaßt, wird der Wein aufgebunden und bespritzt, werden Gemüsekulturen veredelt und Straßen gebaut. Rationell wird der Dünger geschichtet, gewisse Pflanzen werden in Holzkisten gezogen, das Wachstum jeder Sorte Getreide wird studiert. ${ }^{51}$

Immer wieder stellt sich die Frage, ob das von Schalek Festgehaltene nur als Propaganda zu bewerten ist. Dass man im Krieg kämpft, verwundet wird, versorgt werden muss und fällt, ist bekannt. Weiß man aber auch, dass man jeden Tag leben muss, um zu überleben, dass Essen, Trinken, Sekretieren, Schlafen und zum Zahnarzt gehen zu den normalsten alltäglichen Verrichtungen gehören, die der Krieg zur Un-Normalität degradiert hatte? Die Zivilisten daheim, aber auch noch die späteren Generationen daran zu erinnern, war Schalek ein wichtiges Anliegen.

\section{Schalek in der slowenischen Presse}

Im slowenischen ethnischen Gebiet war Alice Schalek nicht unbekannt. Und auch hier polarisierten ihre Artikel die Öffentlichkeit genauso wie in der Haupt- und Residenzstadt. Nach dem heutigen Forschungsstand wurden die Leser hierzulande zum ersten Mal auf die Weltreisende Schalek in »Die Südmark. Blätter zur Unterhaltung und Belehrung für Haus und Familie« (Sonntagsbeilage der »Deutschen Wacht in Cilli«) aufmerksam. Bereits vor 136. Ihre Großmutter hatte in einer Schokoladenfabrik in Bruck an der Leitha gearbeitet und erinnerte sich an diese Zeit als an einen guten Zeitabschnitt ihres Lebens.

50 Popov: Sledi časa.

51 Schalek: Bei der Isonzoarmee. Zum Monte Cosich, dem südlichen Frontfpeiler an der Adria, S. 4. 
dem Ersten Weltkrieg hatte man in einer Notiz der Rubrik» Vermischtes «, 52 unter Berufung auf einen Beitrag von Schalek im Januarheft von »Westermanns Monatsheften «, auf den Unterschied zwischen indischen Tänzerinnen und einer Bajadere hingewiesen. Weswegen man sich ausgerechnet in Celje für indische Tänzerinnen und Bajaderen bzw. für Alice Schalek interessiert hatte, bleibt trotz des verbürgt großen Interesses der Cillier für Reiseberichte im Allgemeinen offen. ${ }^{53}$

Zum zweiten Mal wird Alice Schalek in einer slowenischen Zeitung im Rahmen ihrer Tätigkeit für das Schwarz-gelbe Kreuz erwähnt, und zwar in der Triester Edinost $« .{ }^{54}$ Am gleichen Tag druckte auch die »Laibacher Zeitung « auf der ersten Seite eine praktisch gleich lautende Notiz zur Tätigkeit des Schwarz-gelben Kreuzes: Die Gemahlin des Statthalters Baronin Bienerth, lesen wir in den beiden Blättern, überreichte dem Bürgermeister von Wien, Dr. Weiskirchner

am Vortage des Namenstages Seiner Majestät des Kaisers [...] einen Betrag von 100.000 Kronen für die monatliche Freitischablösung und einen Betrag von 100.000 Kronen als Erlös der Aktion des Schwarz-gelben Kreuzes. Die dem Bürgermeister übergebene Summe gewährt die Möglichkeit, während der Dauer eines Monates, also bis Anfang November, täglich 33.333 Bedürftigen eine warme Mittagskost zu bieten. ${ }^{55}$

In beiden Notizen findet die Komitee-Dame Alice Schalek als Anregerin des Schwarz-gelben Kreuzes Erwähnung.

Am 25.12.1915 wird in »Edinost« ihre Kurzgeschichte zu Kotor wiedergegeben. Hervorgehoben werden hier vor allem die Treue der lokalen Bevölkerung zu Österreich und die Normalität des Lebens trotz des Krieges. Auch ein Befehl des Königs Nikita findet darin Erwähnung, wonach die montenegrinischen Soldaten nur dann auf die österreichischen Kräfte zu schießen hatten, wenn diese als erste das Feuer auf sie eröffnen würden. ${ }^{56}$

Am 4.6.1916 erscheint in »Edinost«, wohl wegen der Nähe zur Front, ein langer Bericht Schaleks über die Kämpfe am Isonzo, in Monte Sabotino, Sveta Gora, Oslavje und Podgora. Das besondere Augenmerk Schaleks wird in diesem übersetzten Artikel, für die heutigen Leser nicht uninteressant, auf italienische Gegenpropaganda gerichtet. ${ }^{57}$ Auch später noch bleibt man

53 Die 15 Jahre jüngere und später ebenfalls weltberühmt gewordene Weltreisende aus Celje, Alma Maximiliane Karlin (1889-1950), begann ihre Reportagen erst zwischen den beiden Kriegen in ihrer Geburtsstadt zu veröffentlichen.

54 Kor.: Črno-rumeni križ za prehrano revežev, S. 1.

55 Anonym: Kriegsfürsorge.

56 Anonym: Kako je v Kotoru, S. 2.

57 Anonym: S soške fronte, S. 2. 
in »Edinost« Alice Schalek treu. So berichtet man am 16. November 1916 über die »interessanten Eindrücke und Beobachtungen der Kriegsberichterstatterin Alice Schalek von der `Neuen Freien Presse` von der montenegrinischen Kampflinie und der Kampf-Charakteristik der Montenegriner «. ${ }^{58}$ Die Montenegriner werden mit sehr viel Sympathie als wendige, mutige, tapfere Soldaten dargestellt, die vor feindlichen Waffen keine Angst haben, allerhöchstens vor den Maschinengewehren, die sie »Schwabenmusik «nennen. ${ }^{59}$ Die Montenegriner, so der Artikel, würden sich beim Schießen hinter den Steinen verstecken, da das Ausheben von Kampfgräben in steinerner Erde zu schwierig sei. Ihre Opfer, ob Verletzte oder Tote, würden sie nie auf dem Kampffeld zurücklassen, im Unterschied zu den Italienern am Isonzo. Die Montenegriner hätten ihre Freude über den Fall von Przemysl im Tanz (»Kolo« $)^{60}$ zum Audruck gebracht, während sie die serbischen Verluste in Belgrad bitter beklagt hätten: „Švabo, Švabo, po boga, što radiš sa našim Srbima? ${ }^{61}$ Der montenegrinischen Frau, die das Heer mit Nahrung versorgt und mitunter sogar den Mann für eine kurze Zeit, in der er sich zuhause stärken kann, an der Front ersetzt, wird im Artikel ein Denkmal gesetzt.

Auch das amerikanische Blatt für Slowenen "Clevelandska Amerika«, das montags, mittwochs und freitags erschien, zeigte Interesse an der Berichterstattung Schaleks. Es brachte am 7.2.1916 einen Bericht, der über Genf an die Redaktion gelangte und unter dem Titel Österr. Verwüstungen an der herzegowinischen Grenze zu Schaleks Artikel aus der »Neuen Freien Presse« vom 11.12.1915 Stellung nahm. Darin hätte Schalek, so der Kommentator, in erster Linie das Elend der Menschen und des Landes thematisiert. ${ }^{62}$

Mit einem ihrer Isonzo-Vorträge kam Schalek während des Kriegs auch nach Ljubljana, ins Hinterland der Isonzo-Front. Am 30.10.1916 wurde in der »Laibacher Zeitung « eine große Anzeige veröffentlicht, in der man ihren Vortrag Drei Monate an der Isonzofront für »heute um 9 Uhr abends im >Kino Central im Landestheater « ankündigte. ${ }^{63}$ Bereits eine Woche später wurden in der Rubrik »Lokal- und Provinzial-Nachrichten « die Leser darüber informiert, dass »[d]ie Leitung des Kino Central [...] dem Stadtmagistrate den Reinertrag des Vortrages der Alice Schalek im Landestheater im Betrage

Ebd.

60 Ebd.

61 Ebd. In deutscher Übersetzung: »Schwabe, Schwabe [pejorativ für Deutscher], um Gottes willen, was tust du unseren Serben an?«

62 Anonym: Avstr. pustošenje ob hercegovski meji, S. 2.

63 »Laibacher Zeitung« (30.10.1916), S. 1773. 
von $500 \mathrm{~K}$ zu Gunsten armer Familien gefallener Soldaten übermittelt« habe. ${ }^{64}$ Interessanterweise findet sich in der »Laibacher Zeitung« kein Bericht über ihren Vortrag. Dafür wird aber ein halbes Jahr darauf, am 23.4.1917, in dieser Zeitung die Information der »Wiener Zeitung« abgedruckt, wonach der Kaiser Alice Schalek das Goldene Verdienstkreuz mit der Krone am Bande der Tapferkeitsmedaille verliehen habe:

Die Kriegsberichterstatterin Alice Schalek gehört als einzige österreichische Schriftstellerin seit Juli 1915 dem k.u.k. Kriegspressequartier an und hat viele Monate in den vordersten Linien der Tiroler, der Isonzo- und der montenegrinischen Front zugebracht. Die Früchte dieser mit vielfacher Gefahr des eigenen Lebens verbundenen Arbeit sind zwei Kriegsbücher: »Tirol in Waffen« und »Am Isonzo«, beide mit eigenen Aufnahmen illustriert, und der Vortrag "Drei Monate an der Isonzo-Front « mit 220 eigenen Bildern. Für die Überlassung vieler Hunderter ihrer Aufnahmen aus dem Feuerbereich an das Kriegsarchiv hat Alice Schalek bereits eine belobende Anerkennung gefunden. Der Vortrag ist $21 \mathrm{Mal}$ in Deutschland und 21 Mal in Österreich vor etwa 26.000 Zuhörern gehalten worden und hat durch seine tiefe Wirkung dazu beigetragen, den Ruhm unserer Isonzo-Armee zu verbreiten. In unserer Stadt fand der Vortrag am 30. Oktober d. J. im Landestheater statt. ${ }^{65}$

Unter den politischen Nachrichten im Laibacher slowenischen Konkurrenzblatt »Slovenski narod « war am 29.11.1917 ein gehässiger Artikel zur »Sonderberichterstatterin aus dem Schlachtfeld « zu lesen. ${ }^{66}$ Schalek hätte, so der Grundtenor, in ihren Artikeln nicht nur detailgetreue Beschreibungen von Kampffeldern und -ereignissen geliefert, sondern auch Vorträge darüber in diversen Orten gehalten, wie z.B. in Ljubljana und Inomost (slowenisch für Innsbruck), weswegen einige Abgeordnete beim Landwehrminister interveniert hätten. Sie sei eine sensationslustige Dame, die beim Sterben mutiger Soldaten aus Abenteuerlust dabei sein musste, um ihren niedrigsten Instinkten frönen zu können. Aus dem gleichen Grund hätte man diesem emanzipierten »Unweib ${ }^{67}$ die Möglichkeit gegeben, zahlreiche Fotos von Leichen zu schießen und in diversen Städten Vorträge zur Lage in den Schießgräben und bei den Erkundungsoffizieren zu halten. Die Abgeordneten hätten wegen ihrer Tätigkeit den Schutz der Soldaten verlangt. ${ }^{68}$ Der Einfluss von Karl Kraus ist in diesem Artikel unverkennbar.

Das samstags erscheinende Wochenblatt »Jugoslovan« (10.11.1917 bis 13.7.1918), dessen verantwortlicher Redakteur der Landesabgeordnete

65 Auszeichnung der Kriegsberichterstatterin Alice Schalek, S. 600.

66 Anonym: Alice Schalek (29.9.1917), S. 3.

67 Die slowenische Zeitung übernimmt hier offensichtlich Karl Kraus' Beschimpfung der Alice Schalek. 
(Josip) Jože Gostinčar (1860-1942) war, ${ }^{69}$ nahm sich der südslawischen Frage mit besonderem Nachdruck an. In diesem Zusammenhang ist wohl auch ein Beitrag zu verstehen, der in der ersten Nummer in der Rubrik »Glasovi« (Stimmen) die Theatersaison im »hübschen « ${ }^{70}$ deutschen Theater von Ljubljana bespricht, das vom »fremdländisch $\aleph^{71}$ (gemeint ist Deutsch) sprechenden Publikum besucht werde. Doch sei das Theater, so das Blatt, ob der Unterhaltungsqualität der Veranstaltungen nicht zu beneiden, denn diese sei auf Grund von fragwürdigen Aufführungen mehr als zweifelhaft. Hinzu käme noch, dass das slowenische Landestheater als das einzige in diesem Gebiet schon länger geschlossen sei, da man für seine Tätigkeit weder Licht noch Heizung erübrige. Noch schlimmer sei es, dass darin der allseits stark kritisierte Kinematograf gastiere, welcher in Ermangelung anderer Zerstreuungen ständig ausverkauft sei. Hier würden auch Veranstaltungen abgehalten, in welchen man, wie von der Zeitung »Slovenec « regelmäßig berichtet, »naturgemäß« in deutscher Sprache vortrüge. Dieses >naturgemäß

[...] erklärt uns auch, wie es möglich ist, dass zu einem Vortrag in das slowenische Landestheater die Korrespondentin des führenden jüdischen Wiener Sprachrohrs eingeladen wurde, eines Blattes der Geldmagnaten, Kriegsspekulanten und Spekulanten mit unserem Blut, das Sprachrohr der Kriegslieferanten und -hetzer; jene Schalek, die derart wild schreibt und vorträgt, dass christlich-soziale Bauernabgeordnete am Ministerium für Landeswehr eine Interpellation gegen sie eingereicht haben, in welcher man sie »emanzipiertes Unweib « und »an seinen primitivsten Instinkten irregewordenes Weib «nennt. Jenes "naturgemäß « erklärt uns auch, wieso die Schalek aus der slowenischen Hand einen Strauß entgegennahm [...] zum Dank dafür, dass sie bei uns die Unkultur zu verbreiten hilft. ${ }^{72}$

Das prosüdslawische Blatt erwies sich hier als antisemitisch, antideutsch und antikapitalistisch.

Auch nach dem Krieg schien Schalek in slowenischen Gebieten nicht ganz vergessen worden zu sein. Sieben Jahre nach Kriegsende wurde in der Görzer slowenischen Publikation »Goriška straža«, die zweimal wöchentlich erschien, auf der vorletzten Seite der Artikel Was gibt es Neues auf dem Lande? veröffentlicht, signiert von den »Betroffenen«. Auf der Wirtschaftsseite des Blattes schneidet der Beitrag ein brisantes Thema an, sich dabei auf Schalek berufend. ${ }^{73}$ Die Bauern aus den im Krieg durch Kämpfe und Gasanwendung stark devastierten Dörfern Pevma und Oslavje, deren Häuser »zur Straßenverbesserungen« verwendet, deren Weinberge

71 Ebd.

72 Ebd. Übersetzung M. M. Z.

73 Prizadeti: Kaj je novega na deželi, S. 3. 
zerstört wurden und deren Erde tot war, worüber Schalek in Wiener Zeitungen berichtet habe, kämen auf Einladung des Gemeinde-Kommissars zusammen, um zu erfahren, dass man das einstige Landgut Fogars in Pevma unter günstigen Bedingungen an Kleinbesitzer zu veräußern trachte. Die Betroffenen wollten es kaufen, um ihre Landwirtschaft zu erneuern. Inzwischen habe es sich jedoch herausgestellt, dass nicht die Kleinbesitzer die Käufer werden sollten, sondern die großkapitalistische Firma Brunner, die bereits zahlreiche Arbeiter in Podgora versklavt hätte. Im Bericht wird außerdem über hohe Steuern geklagt und darüber, dass man immer noch an Lebensmittelmangel leide. Die Betroffenen fordern infolge dessen sowohl die Landesverwaltung in Udine als auch den Staat auf, ihnen bei der Organisation eines weiteren Treffens zu helfen und ihnen Bescheid zu geben, woran sie in Sachen Ankauf seien.

Am 11.11.1927 wurde in der Zeitung "Jutro« eine Anzeige der Volkshochschule Maribor veröffentlicht, wonach dort am »Montag, dem 14. November um 8 Uhr abends die Wiener Autorin und Weltreisende Fr. Al. Schalek einen Vortrag >über Sumatra als dem schönsten Land der Welt $\triangleleft$ halten wird auf Grund von wunderschönen kolorierten skioptischen Bildern. « ${ }^{74}$ Die »Marburger Zeitung « vom 17.11.1927 lobte diesen Vortrag von Schalek folgendermaßen:

Am Montag abends hielt die bekannte Weltreisende und Journalistin Alice Schalek (Wien) einen formvollendeten und hochinteressanten Lichtbildervortrag über Sumatra. Die Vortragende wußte persönlich Erlebtes mit herrlichen, eigenen Aufnahmen aus dem "Schönsten Land der Erde« derart fesselnd zu verquicken, daß das Publikum ihr mit nichtendenwollendem Beifall die Befriedigung über den genußreichen Vortragsabend dankte. Der dichtgefüllte Vortragssaal bewies, daß derartige Vorträge dem Kulturhunger der Draustadt voll und ganz entsprechen. ${ }^{75}$

Dass Schalek im Krieg von den Isonzofronten berichtet hatte, findet im letzten Beitrag zu Schalek, der in Slowenien veröffentlicht wurde, keine Erwähnung mehr.

\section{Abschließend}

Ihre zwanzig Berichte zu den Isonzo-Schlachten ließ Schalek folgendermaßen ausklingen:

Daß ich selbst todmüde bin - fast zwölf Stunden sind wir unterwegs - und daß mir in dieser erbarmungslosen Sonnenstrahlung der Wiederaufstieg auf den Bodil reichlich 
sauer fällt, ist selbstverständlich. Und so macht es mir den weit tieferen Eindruck, daß auch der Sappeurhauptmann, der mit uns geht, kaum mehr weiter kann. Tagtäglich geht er denselben Weg. Heute nacht hat er des Minenganges halber nicht geschlafen. Die Kräfte reichten gerade noch für den Abstieg aus. Jetzt ist es zu viel. Es ist der letzte Eindruck von der Isonzofront. Das Bild des Mannes nehme ich fort, das symbolische Bild eines der tausend Wächter vom Isonzo, die bis zum letzten Kräfteaufgebot auf ihrem Posten stehen. ${ }^{76}$

Nach dem Krieg wurde Schalek zur Kriegskritikerin. Ihre Rolle im Krieg sah sie als die einer Beobachterin, die am Ganzen keine Schuld trug. Dies mag wohl wahr gewesen sein. Andererseits stimmt es, dass ihre gekonnt geschriebenen Beiträge auch davon zeugen, dass sie zwar nicht vom Krieg, vielmehr aber von den kämpfenden Soldaten und Offizieren fasziniert war und ihre Taten geschildert hatte, ohne dabei zu diesem Zeitpunkt zu einer besonders scharfen Kriegskritikerin oder gar -gegnerin geworden zu sein.

Als nach dem Zerfall der Donau-Monarchie der neue südslawische Staat entstand, gab es dort keine Vergangenheitsaufarbeitung: Die Slowenen und Kroaten gehörten zu den Verlierern, die Serben zu den Gewinnern des Großen Krieges. So einfach war das. Man schwieg sich an und aus. Daher widme ich nun, mehr als 100 Jahre nach dem Ausbruch des Ersten Weltkrieges, meinen Beitrag zu Alice Schalek, die den Soldaten am Isonzo eine Stimme gab, meinen Großvätern: Ivan Bajt (1885-1973), der am Isonzo kämpfte und vom ganzen Krieg nur das zu berichten bereit war, dass es schlimm war und dass Doberdob zum Grab junger slowenischer Männer wurde; dem Saloniki-Kämpfer Svetozar Miladinović (1882-1963), der in die Balkankriege zog, um Ende 1918, nach sechs Jahren Abwesenheit, heimzukehren und seine lebenserhaltenden Kriegserfahrungen später an alle drei Söhne, die im Zweiten Weltkrieg kämpften, einer bei den Partisanen, zwei bei den Tschetniks, weiterzureichen; und an Ciril Zalaznik (1900-1983), der über seine Einsätze am Isonzo überhaupt nie etwas gesagt hatte. Ich denke, sie hätten nichts dagegen, sich hier vereint (wieder) zu finden.

\section{Literaturverzeichnis}

Alice Schalek am Vortragstisch der Volkshochschule. »Marburger Zeitung«(17.11.1927), S. 4. Anonym: Alice Schalek. »Slovenski narod « (3.11.1916).

Anonym: Alice Schalek. »Slovenski narod« (29.9.1917), S. 3.

Anonym: Avstr. pustošenje ob hercegovski meji. »Clevelandska Amerika« (7.2.1916), S. 2. Anonym: Glasovi. Gledališka sezona. »ugoslovan« (10.11.1917), S. 2f.

Anonym: Kako je v Kotoru. »Edinost« (25.12.1915), S. 2. 
Anonym: Kriegsfürsorge. »Laibacher Zeitung« (4.10.1914).

Anonym: Ob črnogorski meji. »Edinost « (16.11.1915), S. 2.

Anonym: S soške fronte. »Edinost« (4.6.1916), S. 2.

Anonym: [o. T.]. »Die Südmark. Blätter zur Unterhaltung und Belehrung für Haus und Familie. Sonntagsbeilage der 〉Deutschen Wacht $<$ in Cilli« 2 (14.11.1911), S. 4.

Auszeichnung der Kriegsberichterstatterin Alice Schalek. »Laibacher Zeitung (23.4.1917), S. 600 .

Brk. [Brenk], L[an]: Gostinčar, Josip. In: Enciklopedija Slovenije. Bd. 3. Hgg. Marjan Javornik et al. Ljubljana: Mladinska knjiga 1989.

Hamann, Brigitte: Bertha von Suttner. Ein Leben für den Frieden. München, Zürich: Piper Taschenbuch ${ }^{3} 2006$.

Kirschstein, Daniela: Writing War. Kriegsliteratur als Ethnographie bei Ernst Jünger, LouisFerdinand Céline und Curzio Malaparte. Würzburg: Verlag Königshausen \& Neumann 2014.

Kor.: Črno-rumeni križ za prehrano revežev. »Edinost « (4.10.1914), S. 1.

Kraus, Karl: Die letzten Tage der Menschheit. Tragödie in fünf Akten mit Vorspiel und Epilog. In: ders.: Schriften. Bd. 10. Hg. Christian Wagenknecht. Frankfurt/M.: Suhrkamp 1989.

Kraus, Karl: Ferdinand und die Talente. »Fackel« 16.400-403 (10.7.1914), S. 1-4.

Kraus, Karl: Notizen. »Fackel« 18.423-425 (5.5.1916), S. 17-19.

Kraus versus Schalek. Es wird scharf geschossen. "DiePresse.com« (28.6.2013). <http:// diepresse.com/home/165jahre/1424146/Kraus-versus-Schalek_Es-wird-scharfgeschossen> (Zugriff 21.5.2015).

Lughofer, Johann Georg: „Der Pazifismus ist geradezu der Überwinder des nationalen Chauvinismus «. Die Waffen nieder! aus interkultureller Perspektive. In: Im Prisma: Bertha von Suttner: »Die Waffen nieder!«. Hg. Johann Georg Lughofer. Wien, St. Wolfgang: Edition Art Science 2010, S. 169-191.

Ljudska univerza v Mariboru. »Jutro« (11.11.1927), S. 5.

Miladinović Zalaznik, Mira: "Ich hab's gewagt [...] ich hab's gesagt «! Bertha von Suttners Die Waffen nieder! In: Im Prisma: Bertha von Suttner: »Die Waffen nieder!«. Hg. Johann Georg Lughofer. Wien, St. Wolfgang: Edition Art Science 2010, S. 157-167.

Popov, Jurij: Sledi časa. Sto let začetka soške fronte. Radiosendung. Radio Slovenija 1 (24.5.2015). <http://4d.rtvslo.si/arhiv/sledi-casa/174337589> (Zugriff: 25.5.2015).

Prizadeti: Kaj je novega na deželi. »Goriška straža« (22.1.1925), S. 3.

Rauchensteiner, Manfried: Der Erste Weltkrieg und das Ende der Habsburgermonarchie 1914-1918. Wien, Köln, Weimar: Böhlau Verlag 2013.

Schalek, Alice: Am Isonzo. März bis Juli 1916. Wien: L. W. Seidel \& Sohn 1917.

Schalek, Alice: Bei der Isonzoarmee. Ankunft im Hauptquartier. "Neue Freie Presse«, Morgenblatt (4.4.1916), S. 1.

Schalek, Alice: Bei der Isonzoarmee. Auf den Tolmeiner Brückenkopf, den Mrzli Vrch und Bodil Vrch. »Neue Freie Presse«, Morgenblatt (15.9.1916), S. 1-4.

Schalek, Alice: Bei der Isonzoarmee. Aufstieg zum Krn. »Neue Freie Presse«, Morgenblatt (19.8.1916), S. 1-4.

Schalek, Alice: Bei der Isonzoarmee. Das Geheimnis der Podgora. "Neue Freie Presse«, Morgenblatt (20.4.1916), S. 1-2.

Schalek, Alice: Bei der Isonzoarmee. Die Kote von Plava. »Neue Freie Presse«, Morgenblatt (27.7.1916), S. 1-4.

Schalek, Alice: Bei der Isonzoarmee. Ein Angriff über die Tolmeiner Brücke. »Neue Freie Presse«, Morgenblatt (10.8.1916), S. 1-4. 
Schalek, Alice: Bei der Isonzoarmee. Eine Mondnacht auf dem Monte Sabotino. »Neue Freie Presse«, Morgenblatt (14.4.1916), S. 1-4.

Schalek, Alice: Bei der Isonzoarmee. In Görz. »Neue Freie Presse«, Morgenblatt (7.4.1916), S. $1-4$.

Schalek, Alice: Bei der Isonzoarmee. Nach San Martino del Carso. »Neue Freie Presse«, Morgenblatt (13.7.1916), S. 1-4.

Schalek, Alice: Bei der Isonzoarmee. Oslavija, der gestorbene Hügel. »Neue Freie Presse«, Morgenblatt (24.5.1916), S. 1-5.

Schalek, Alice: Bei der Isonzoarmee. Von der Front in die Etappe. "Neue Freie Presse«, Morgenblatt (17.5.1916), S. 1-3.

Schalek, Alice: Bei der Isonzoarmee. Von Görz nach Doberdo. »Neue Freie Presse«, Morgenblatt (31.5.1916), S. 1-4.

Schalek, Alice: Bei der Isonzoarmee. Zum Monte Cosich, dem südlichen Frontfpeiler an der Adria. Neue Freie Presse«, Morgenblatt (20.7.1916), S. 1-4.

Schick, Paul: Karl Kraus in Selbstzeugnissen und Bilddokumenten. Reinbek bei Hamburg: Rowohlt Taschenbuch Verlag 1984.

Štepec, Marko (Hg.): Slovenci + prva svetovna vojna 1914-1918. Ljubljana: Muzej novejše zgodovine Slovenije 2010.

V fokusu: 100 let I. svetovne vojne. Fernsehsendung. Gespräch mit Dr. M. Christian Ortner. TV SLO 1 (7.6.2015).

Wintersteiner, Werner: »Die Waffen nieder! - ein immer noch zeitgemäßes Projekt. In: Im Prisma: Bertha von Suttner: »Die Waffen nieder!«. Hg. Johann Georg Lughofer. Wien, St. Wolfgang: Edition Art Science 2010, S. 193-210.

1914-2014. 100 Jahre erster Weltkrieg [virtuelle Ausstellung des Österreichischen Staatsarchivs]. Darin (Navigation: Frau im Krieg > Alice Schalek > Dokument KA, MBA 543.216): Goldenes Verdienstkreuz mit der Krone am Bande der Tapferkeitsmedaille. $<$ http://wk1.staatsarchiv.at/frau-im-krieg/alice-schalek/\#/?a=artefactgroup401> (Zugriff: 24.5.2015). 